\title{
Cavitation in an Orifice Flow
}

\author{
S. Dabiri* , W. A. Sirignano ${ }^{\dagger}$ \\ Department of Mechanical and Aerospace Engineering, University of California, Irvine, CA 92697 \\ and D. D. Joseph ${ }^{\ddagger}$ \\ Aerospace Engineering and Mechanics Department, University of Minnesota, Minneapolis, MN 55455
}

\begin{abstract}
The purpose of this study is to identify the potential locations for cavitation induced by total stress on the flow of a liquid through an orifice of an atomizer. A numerical simulation of two-phase incompressible flow is conducted in an axisymmetric geometry of the orifice for Reynolds number between 100 and 2000. The orifice has a rounded upstream corner and a sharp downstream corner with length-to-diameter ratio between 1 and 5 . The total stress including viscous stress and pressure has been calculated in the flow field and, from there, the maximum principal stress is found. The total-stress criterion for cavitation is applied to find the regions where cavitation is likely to occur and compared with those of the traditional pressure criterion. Results show that the viscous stress has significant effects on cavitation. The effect of geometry and occurrence of hydraulic flip in the orifice on the total stress is studied. The Navier-Stokes equations are solved numerically using a finitevolume method and a boundary-fitted orthogonal grid that comes from the streamlines and potential lines of an axisymmetric equipotential flow in the same geometry. A level-set formulation is used to track the interface and model the surface tension.
\end{abstract}

\section{Introduction}

High-pressure atomizers and spray generators are of great interest in industry and have many applications such as combustors, drying systems and agricultural sprays.

Recent experimental studies by Tamaki et al. ${ }^{1,2}$ and Hiroyasu ${ }^{3}$ show that the occurrence of cavitation inside the nozzle makes a substantial contribution to the breakup of the exiting liquid jet. Figure 1 shows a schematic of their experiment. The collapse of cavity bubbles can increase the disturbances in the flow leading to a faster breakup of jet. Even with high pressure drops, the main flow of liquid jet does not atomize greatly when disturbances caused by cavitation are not present.

In a different experiment, Otendal et al. ${ }^{4}$ studied the break up of high-speed liquid jet in vacuum, where the pressure is lower than the vapor pressure. By an appropriate design of the nozzle, they avoided the cavitation-induced instabilities inside the nozzle. By decreasing the air pressure below the vapor pressure, they observed a bursting phenomena due to cavitation in the free jet.

Bunnell et al. ${ }^{5}$ studied the unsteady cavitating flow in a slot and found that partially cavitated slots show a periodic oscillation with Strouhal number near unity based on orifice length and Bernoulli velocity.

Tafreshi and Pourdeyhimi ${ }^{6}$ carried out a numerical simulation on cavitation and hydraulic flip inside hydroentangling nozzles. They showed under certain conditions cavity extends to the nozzle outlet and results in hydraulic flip. When hydraulic flip occurs, cavitation vanishes due to the fact that downstream air moves upward into the nozzle. This leads into the elongation of the jet breakup length. Ahn et al. ${ }^{7}$ experimentally studied the effects of cavitation and hydraulic flip, on the breakup of the liquid jet injected perpendicularly into subsonic crossflow. They showed that cavitation results in shortening the liquid column breakup length. They observed smaller breakup length in the hydraulic flip due to the fact that jet diameter was smaller than the orifice diameter. Jung et al. ${ }^{8}$ considered the breakup characteristics of liquid sheets formed by a like-doublet injection. They found that liquid-jet turbulence delays sheet breakup and shortens wavelengths of both ligaments and sheets. Ganippa et al. ${ }^{9}$ considered the cavitation growth in the nozzle

\footnotetext{
*Graduate student researcher, AIAA student member.

$\dagger$ Professor, AIAA Fellow.

$\ddagger$ Professor.
} 
as they increased the flow rate. First, traveling bubbles are created. These bubbles are detached from the wall and move with the stream. By increasing the flow rate, the unsteady cloud of cavitation is observed. Further increasing in the flow rate caused the non-symmetrical distribution of cavitation within the nozzle and its extension to the nozzle exit. More atomization occurs at the side with stronger cavitation.

In the traditional criterion of cavitation, cavitation occurs when the pressure drops below the breaking strength of liquid which in an ideal case is the vapor pressure at local temperature. Joseph ${ }^{10}$ proposed that the important parameter in cavitation is the total stress which includes both the pressure and viscous stress. Therefore, the cavitation occurs when the maximum principal stress drops below the breaking strength of liquid. Using this criterion, Funada et al. ${ }^{11}$ predicted the cavitation of a two-dimensional viscous potential flow through an aperture and Dabiri et al. ${ }^{12}$ studied the cavitation of Navier-Stokes flow in the aperture. Most of the calculations done on the subject of high-pressure-nozzle cavitation have used the traditional criterion. The purpose of this paper is to use the new criterion to study the potential locations for the cavitation in liquid atomizers.

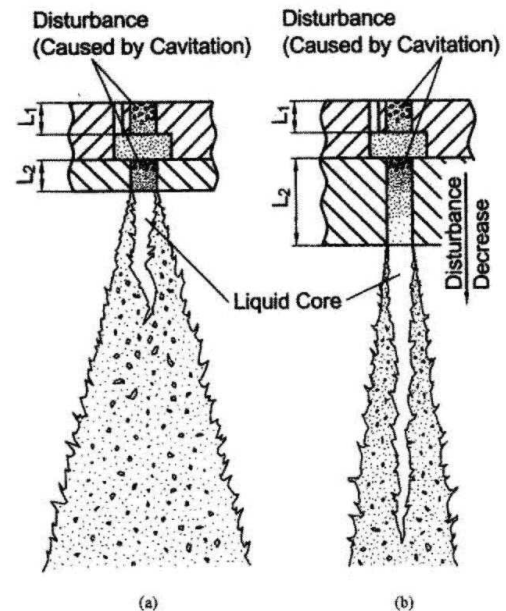

Figure 1: The role of cavitation and the effect of orifice length. ${ }^{2}$

\section{Governing Equations}

In this study, we consider flow of a liquid through an orifice and the resulting exiting jet into a stagnant gas. The physical problem and the computational domain and grid are shown in figure 2 . Governing equations for an unsteady, incompressible viscous flow are the Navier-Stokes equations:

$$
\begin{aligned}
\rho\left(\frac{\partial \mathbf{u}}{\partial t}+\mathbf{u} \cdot \nabla \mathbf{u}\right)= & -\nabla p+\nabla \cdot(2 \mu \mathbf{D})+\sigma \kappa \delta(d) \mathbf{n} \\
\mathbf{D}= & \frac{1}{2}\left[(\nabla \mathbf{u})+(\nabla \mathbf{u})^{T}\right] \\
& \nabla \cdot \mathbf{u}=0
\end{aligned}
$$

where $\mathbf{u}$ is the velocity, and $\rho$ and $\mu$ are the fluid density and viscosity, respectively, which could be properties of either liquid or gas phase. $\mathbf{D}$ is the strain rate tensor. The last term represents the surface tension as a force concentrated on the interface. Here, $\sigma$ is the surface tension coefficient, $\kappa$ is the curvature of interface, $\delta$ is the Dirac delta function. $d$ represents the distance from the interface and $\mathbf{n}$ corresponds to the unit normal vector at the interface. The flow is characterized by the density ratio of gas to liquid, viscosity ratio, and the nondimensional parameters, Reynolds number $(R e)$ and Weber number $(W e)$, which are defined as follows:

$$
R e=\frac{\rho_{l i q} U D}{\mu_{l i q}}, \quad W e=\frac{\rho_{l i q} U^{2} D}{\sigma}, \quad \lambda=\frac{\rho_{l i q}}{\rho_{\text {gas }}}, \quad \eta=\frac{\mu_{l i q}}{\mu_{\text {gas }}}
$$




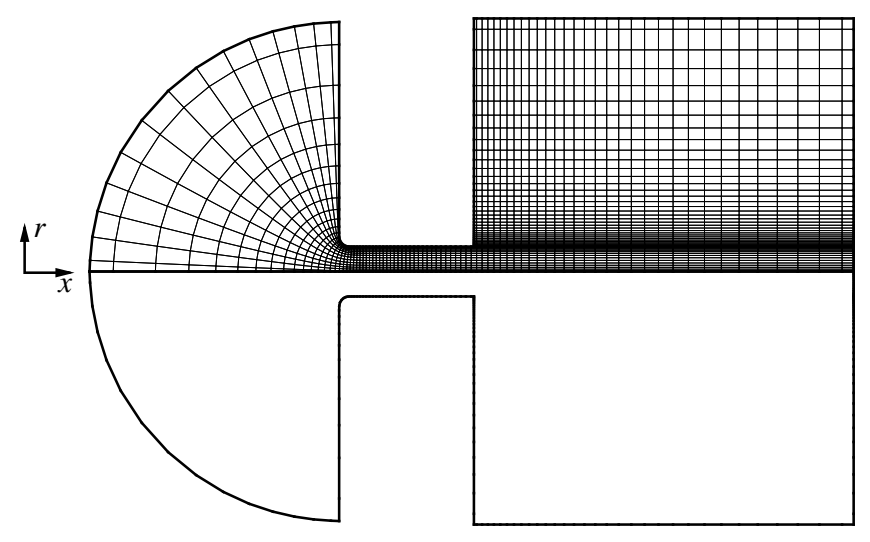

Figure 2: Physical domain and orthogonal grid (flow from left to right)

Here, $D$ is the orifice diameter and $U$ is the theoretical Bernoulli velocity of the jet:

$$
U=\sqrt{\frac{2\left(p_{u}-p_{d}\right)}{\rho_{\text {liq }}}}
$$

where $p_{u}$ and $p_{d}$ are the far upstream and far downstream pressures, respectively.

Finding the velocities and pressure field, the stress tensor is calculated using:

$$
\begin{gathered}
\mathbf{T}=\mu\left[(\nabla \mathbf{u})+(\nabla \mathbf{u})^{T}\right]-p \mathbf{I} \\
p<p_{c}
\end{gathered}
$$

where $\mathbf{I}$ is the identity matrix and superscript $T$ refers to the transpose of a tensor. Therefore, the maximum tensile stress, $T_{11}$ can be calculated by transforming the tensor to the diagonal form.

The new criterion for cavitation proposed by Joseph ${ }^{10}$ is used to find the cavitating regions in the flow field. According to this criterion, cavitation occurs when the maximum principal stress exceeds the negative of the critical threshold stress of liquid at the local temperature:

$$
T_{11}>-p_{c}
$$

The critical threshold stress, $p_{c}$, might be the vapor pressure $p_{v}$ or some other appropriate value. The cavitation number, $K$, defines the critical threshold stress, $p_{c}$, in a nondimensional manner:

$$
K=\frac{p_{u}-p_{d}}{p_{d}-p_{c}}
$$

An orthogonal grid is used to discretize the domain because, in this case, many of the terms in the metric tensor will be zero and the calculation will be faster. Also, it will offer more accuracy in the calculation of the normal fluxes.

Thompson et al. ${ }^{13}$ solved the two-dimensional Poisson equation with an arbitrariness in choice of source terms to generate the orthogonal coordinates. Instead of solving the 2-D Poisson equations, we solve the Laplace equation corresponding to the irrotational axisymmetric flow. Therefore, potential function and stream function of the theoretical axisymmetric potential flow are used as the orthogonal-coordinates system. This choice of coordinates will increase the accuracy since the flow far from the boundaries is irrotational and thus, closely parallel to the grid. Equations for the potential $\phi$ and stream function $\psi$ come from the conditions of potential flow:

$$
\begin{aligned}
\nabla^{2} \phi & =0 \quad \Rightarrow \quad \frac{1}{r} \frac{\partial}{\partial r}\left(r \frac{\partial \phi}{\partial r}\right)+\frac{\partial^{2} \phi}{\partial x^{2}}=0 \\
\omega_{\theta} & =0 \quad \Rightarrow \quad r \frac{\partial}{\partial r}\left(\frac{1}{r} \frac{\partial \psi}{\partial r}\right)+\frac{\partial^{2} \psi}{\partial x^{2}}=0
\end{aligned}
$$


where $\omega_{\theta}$ is the azimuthal component of vorticity. These two equations define the $\phi$ and $\psi$ in the $x-r$ cylindrical coordinates. In a practical case, it is more convenient to solve the equation for $x$ and $r$ in the $\phi-\psi$ coordinates. The inverse form of these equations are derived as follow:

$$
\begin{aligned}
& \frac{\partial}{\partial \phi}\left(\frac{1}{r} \frac{\partial x}{\partial \phi}\right)+\frac{\partial}{\partial \psi}\left(r \frac{\partial x}{\partial \psi}\right)=0 \\
& \frac{\partial}{\partial \phi}\left(\frac{1}{r} \frac{\partial r}{\partial \phi}\right)+\frac{\partial}{\partial \psi}\left(r \frac{\partial r}{\partial \psi}\right)=0
\end{aligned}
$$

These equations are in the same format as derived by Ryskin and Leal. ${ }^{14}$ However, they derived them for a specific choice of source terms in the 2-D Poisson equations, while here, they are derived from equations of irrotational axisymmetric flow. These equations with proper boundary conditions which come from the geometry of the boundaries and Cauchy-Riemann conditions are solved by a second-order, finite-difference code.

\section{Numerical Results}

The numerical solution of the incompressible, unsteady, axisymmetric Navier-Stokes equations is performed using the finite-volume method on a staggered grid. The convective term is discretized using the Quadratic Upwind Interpolation for Convective Kinematics (QUICK) (Hayase ${ }^{15}$ ). The Semi-Implicit Method for Pressure-Linked Equation (SIMPLE), developed by Patankar, ${ }^{16}$ is used to solve the pressure-velocity coupling. The time integration is accomplished using the second-order Crank-Nicolson scheme. The calculation is done for Reynolds-numbers equal to 100, 200, 500, 1000, and 2000. A level-set method is used to track the interface and model the surface tension. Details of the method can be found elsewhere. ${ }^{17,18}$ The level set formulation is benchmarked by the authors in another paper. ${ }^{12}$

The computational domain is shown in figure 2. Upstream and downstream boundaries are at distance of five diameters from the orifice. Pressure boundary conditions are applied on both upstream and downstream boundaries. On the downstream boundary, the Lagrangian time derivative of velocities is set to zero. The grid independency of the solution was investigated for flow with $R e=2000$, by increasing the number of grid points by $50 \%$. The maximum change in the velocity profile at the exit of the nozzle was less than $0.5 \%$. Also, the independency of the solution to the size of the domain is verified. For flow with the $R e=100$ and the domain was extended by $50 \%$, the maximum change in velocity profile at exit was about $0.1 \%$.

\section{A. Effect of Reynolds number on cavitation}

In this section, the axisymmetric flow in a fixed geometry with various Reynolds numbers is studied. Liquidto-gas density ratio and viscosity ratio are $\lambda=100, \eta=10$ respectively, and the Weber number is $W e=1000$ while Reynolds number varies between 100 and 2000. The length-to-diameter ratio of the orifice is $L / D=2$ and the inlet corner is rounded with radius of $r / D=0.02$. However, the outlet corner is sharp.

After several residence time the flow reaches a steady state. It has been observed ${ }^{19,20}$ that the unsteadiness due to Kelvin-Helmholtz or Rayleigh instability will be observable at distances of more then 20 diameters downstream of the orifice. Therefore, in this study we expect to have steady flow everywhere.

For Reynolds number $R e=100$, Figure 3 shows the velocity profiles and normalized pressure distribution. The liquid-gas interface leaving the downstream corner is also shown. The flow has an almost fully developed parabolic profile at the exit and there is no separation at upstream corner. A small contraction can be seen in the liquid jet after leaving the orifice.

The flow for $R e=2000$ is shown in figure 4 . The pressure has a minimum at the upstream corner, where the cavitation is likely to occur. Note that the exit velocity profile indicates a boundary layer but full development has not occurred. At the upstream curved corner the flow separates from the wall and reattaches to the wall further downstream and creates a recirculating region. The recirculating region is shown in figure 4. The recirculating region will grow as the Reynolds number increases.

In the following part the two criteria for predicting the cavitation are compared. For flows with different Reynolds numbers, the total stress is calculated in the flow field and regions where the cavitation occurs based on each criterion is identified. In each plot, the curves in which cavitation is likely to occur is plotted for the same flow parameters but different values of cavitation number, $K$. Figure 5 shows these vulnerable 

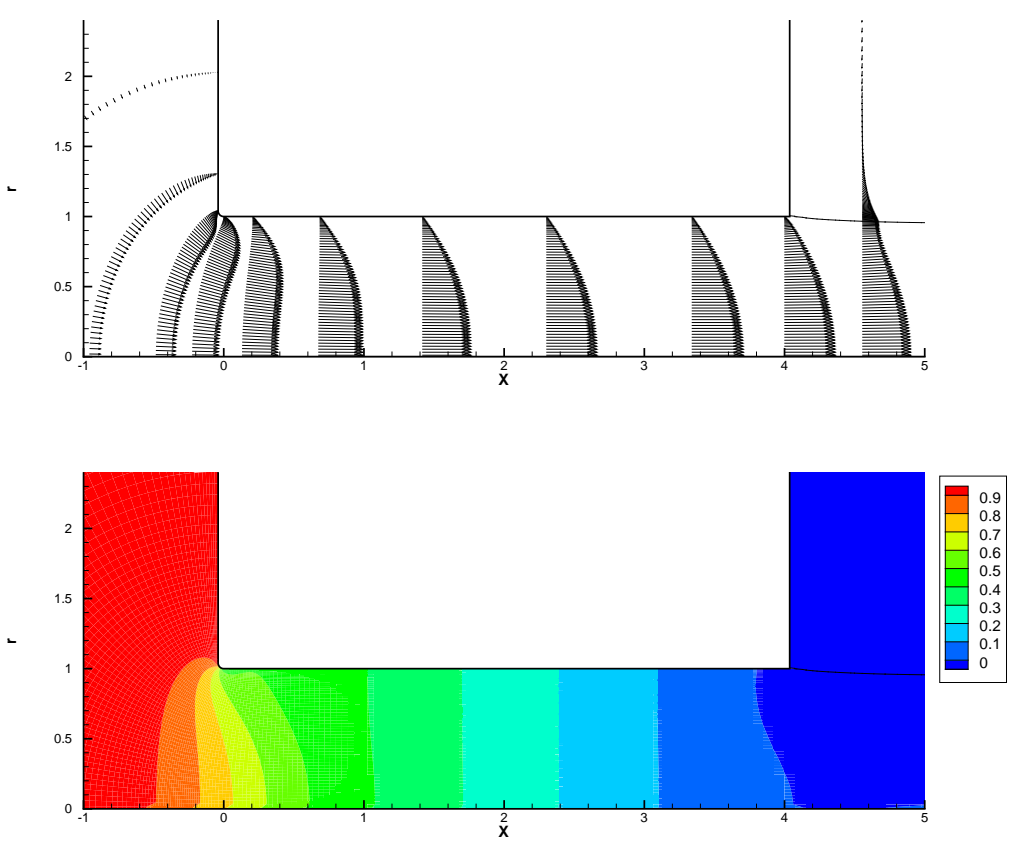

Figure 3: Velocity profiles and contours of $\left(p-p_{d}\right) / \frac{1}{2} \rho U^{2}$ for flow with $R e=100$
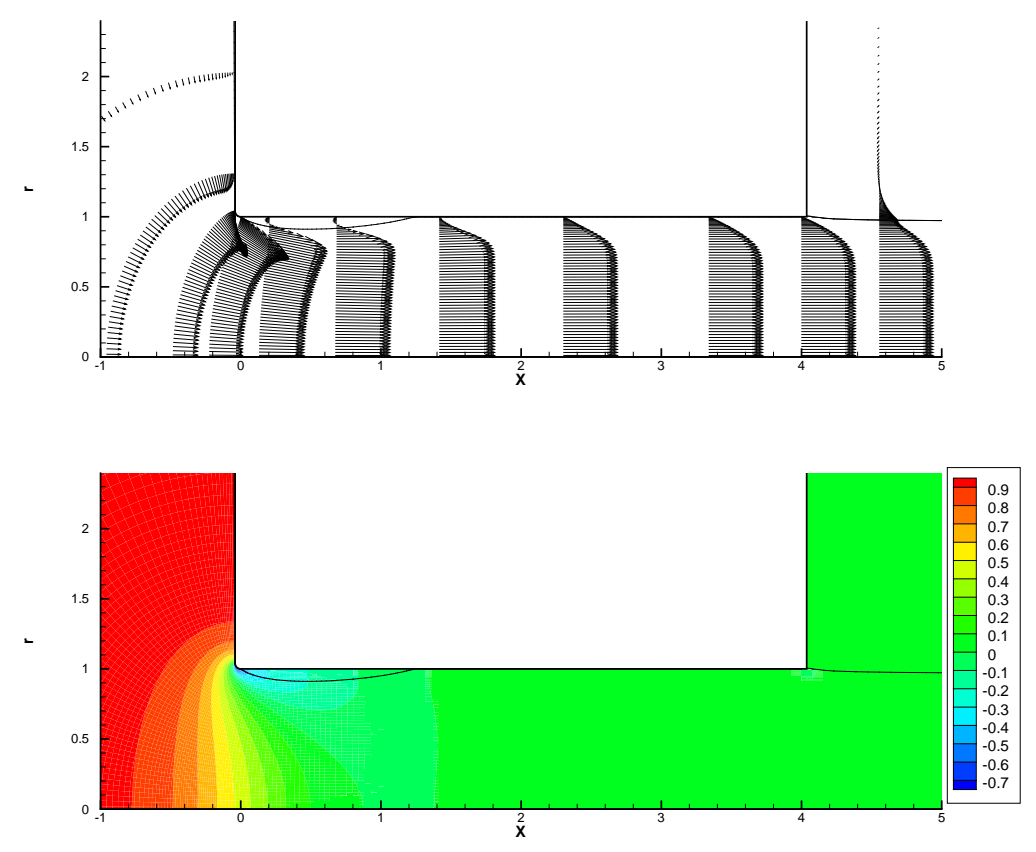

Figure 4: Velocity profiles and contours of $\left(p-p_{d}\right) / \frac{1}{2} \rho U^{2}$ for flow with $R e=2000$ 


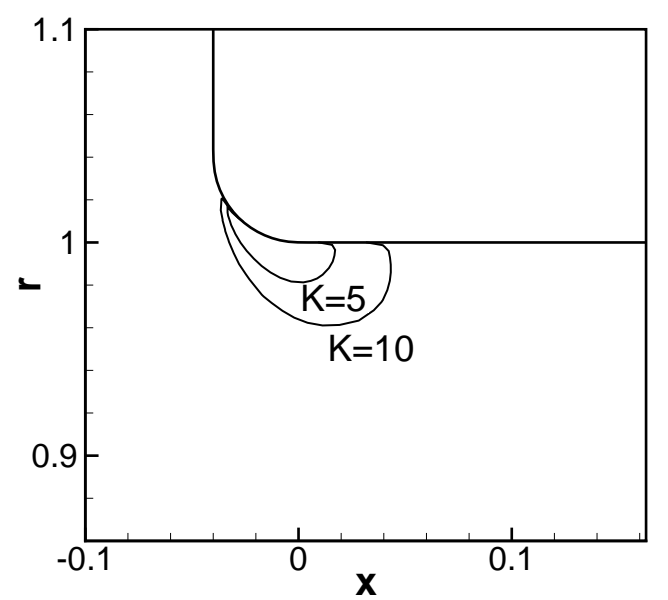

(a) $R e=200$, total stress criterion

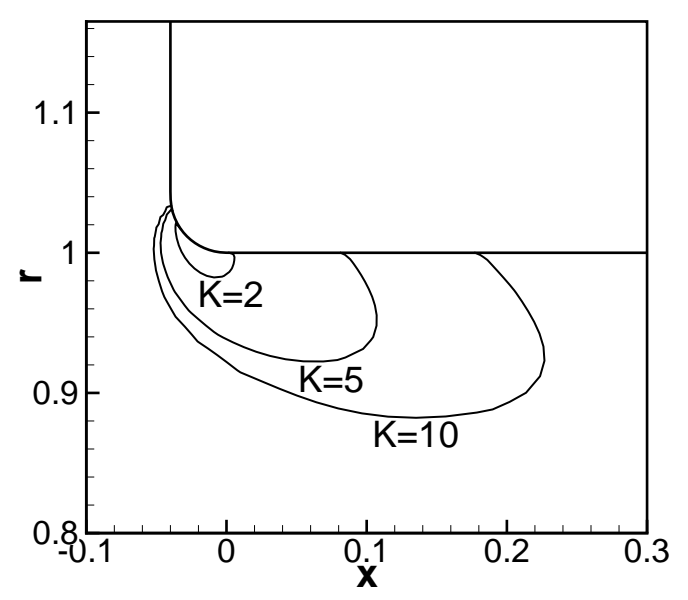

(c) $R e=500$, total stress criterion

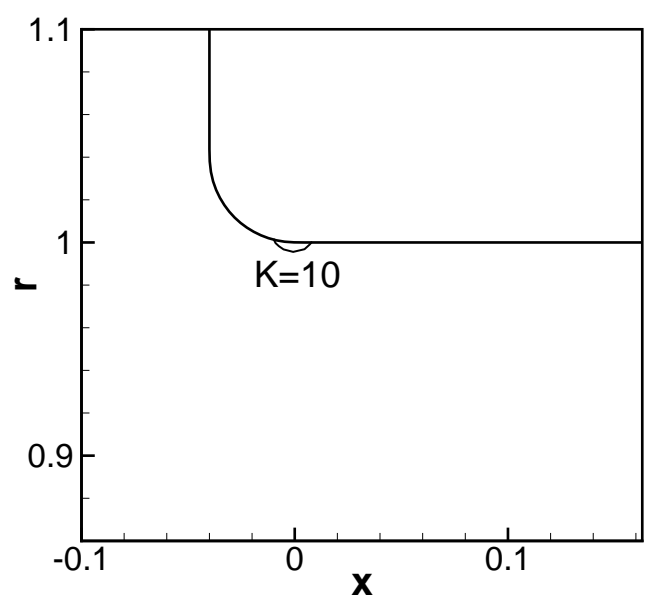

(b) $R e=200$, pressure criterion

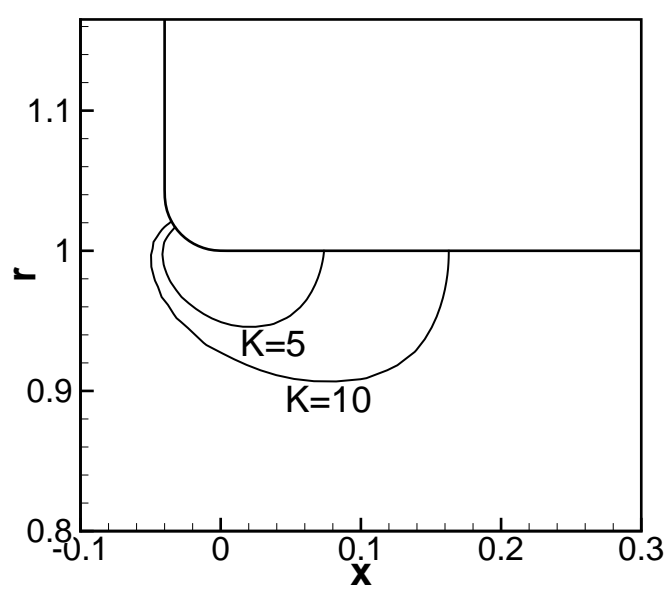

(d) $R e=500$, pressure criterion

Figure 5: The cavitation threshold curves on which $T_{11}+p_{c}=0$ in different flows with $K=2,5,10$. $W e=1000, L / D=1$, (a) $R e=200$, total stress criterion, (b) $R e=200$, pressure criterion, (c) $R e=500$, total stress criterion, (d) $R e=500$, pressure criterion 
regions for $R e=200$ and $R e=500$. At Reynolds number of 200 the pressure criterion predicts almost no cavitation in the flow, while the total stress predicts a larger domain of cavitation. This could be explained by the fact that in the lower Reynolds number the viscous stress is stronger and there will be larger difference between pressure and total stress.

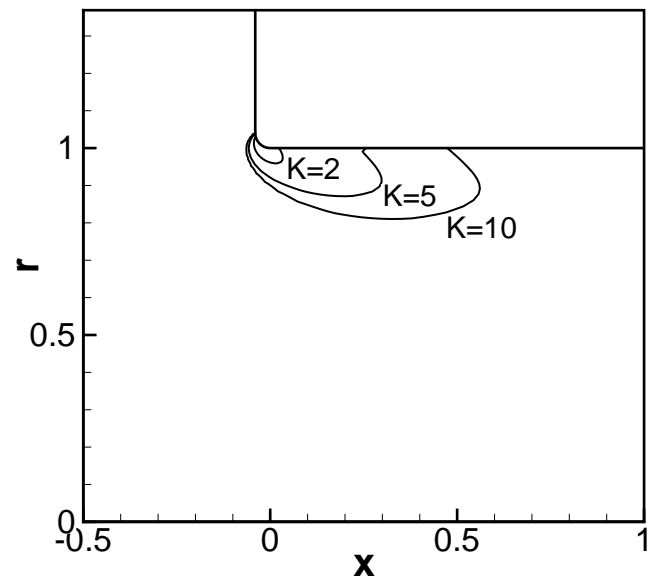

(a) $R e=1000$, total stress criterion

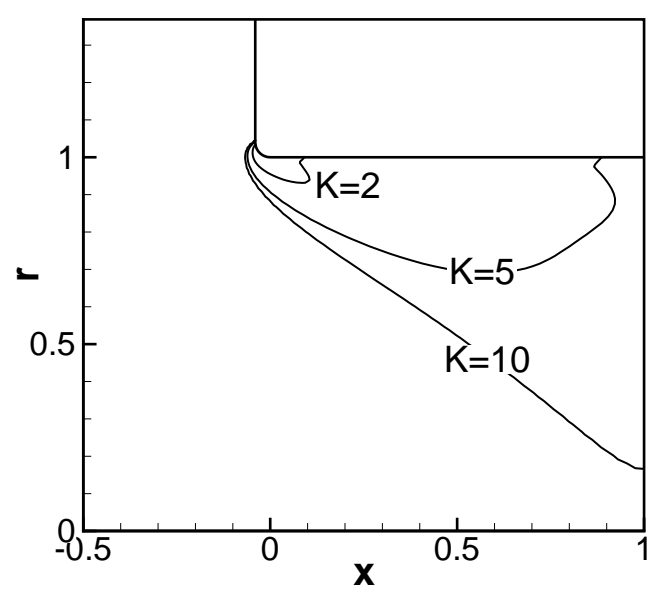

(c) $R e=2000$, total stress criterion

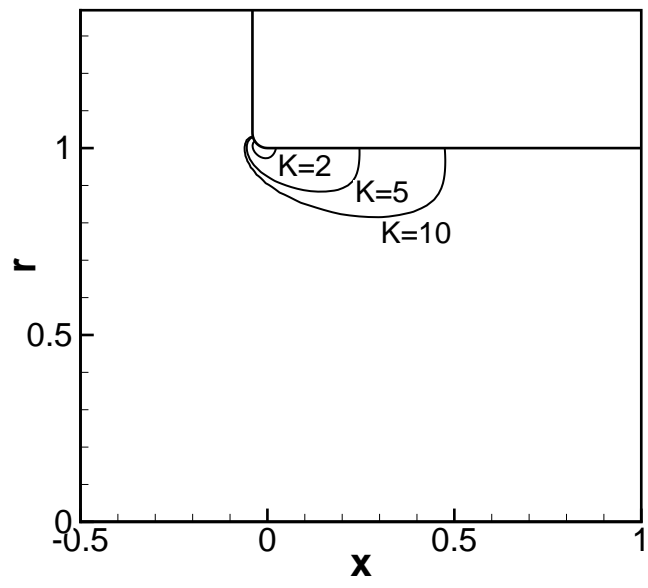

(b) $R e=1000$, pressure criterion

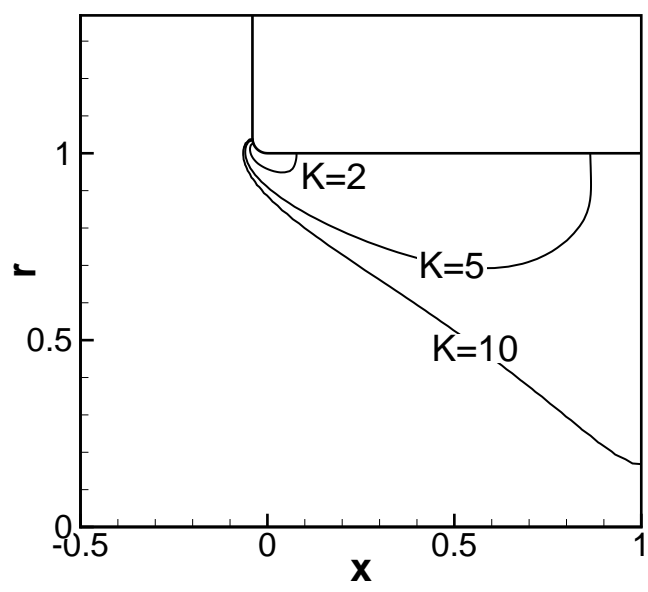

(d) $R e=2000$, pressure criterion

Figure 6: The cavitation threshold curves on which $T_{11}+p_{c}=0$ in different flows with $K=2,5,10$. $R e=2000, W e=1000, L / D=1$, (a) $R e=1000$, total stress criterion, (b) $R e=1000$, pressure criterion, (c) $R e=2000$, total stress criterion, (d) $R e=2000$, pressure criterion

Flows with $R e=1000$ and $R e=2000$ are shown in figure 6. For higher Reynolds numbers the difference between two criteria becomes less but still the cavitating region predicted by the total-stress criterion stretches further downstream. This stretching appears a short distance from the orifice wall. It occurs in the high-shear-stress region inside the separated boundary layer. Note that the plots in figures 5 and 6 have different scales. Figure 7 shows the thresholds values of $K$ above which the cavitation will be present inside the nozzle based on both pressure criterion and total-stress criterion for difference values of length-todiameter ratios. Apparently, the total-stress criterion predicts more chances of cavitation than the pressure criterion. The relative difference between two criteria is larger at lower Reynolds numbers but decreases as the Reynolds number increases. At $R e=2000$ which is close to some practical situations, the relative difference between the threshold value of $K$ predicted by two criteria is about $20 \%, 23 \%$ and $30 \%$ for lengthto-diameter ratios of one, two and five respectively. Going to lower length-to-diameter ratios, two interesting 
thing happen. First is the hydraulic flip which is discussed in section C. The second phnomenon is that for length-to-diameter ratio of $r / D=0.1$, the trend of $K$ based on total-stress criterion versus Reynolds number changes and it increases as Reynolds number increases. This means less chance of cavitation for higher Reynolds number and agrees with the statement by ${ }^{21}$ about the increase in risk of cavitation for more viscous fluids.

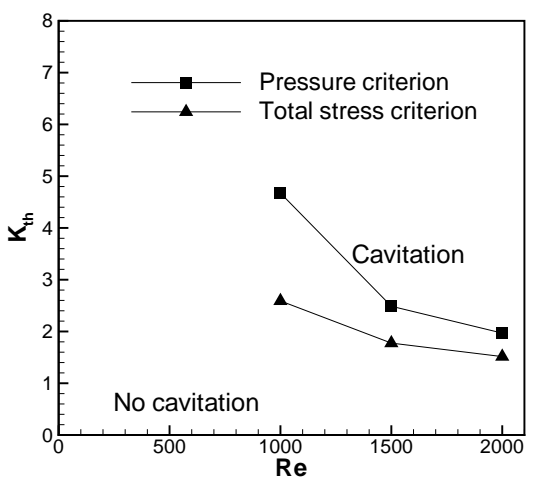

(a) $L / D=5$

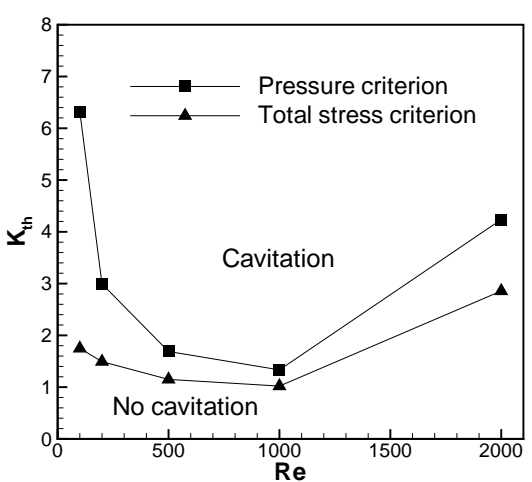

(d) $L / D=0.5$

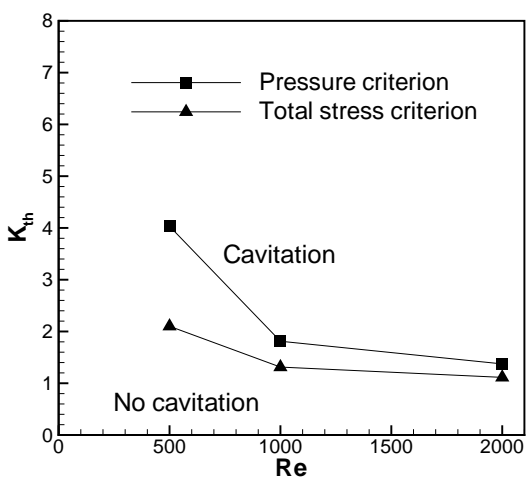

(b) $L / D=2$

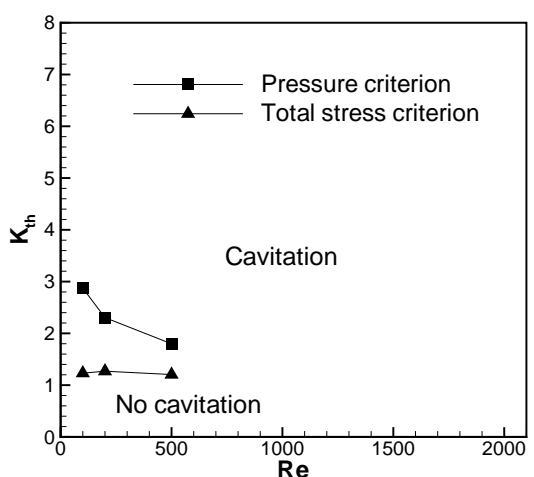

(e) $L / D=0.2$

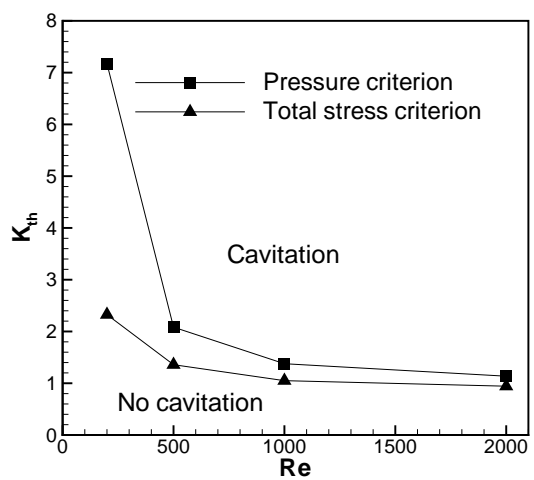

(c) $L / D=1$

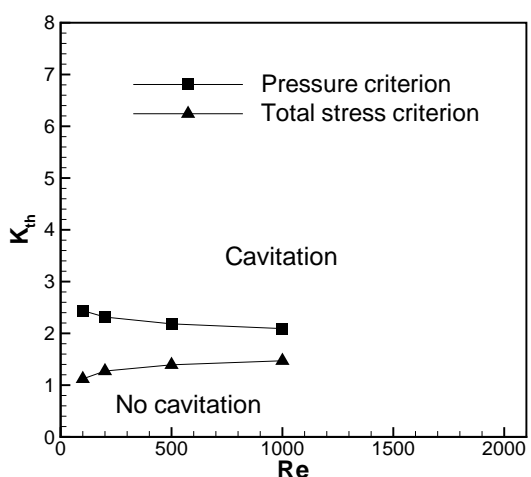

(f) $L / D=0.1$

Figure 7: Threshold values of $K$ above which cavitation occurs in the orifice. (a) $L / D=5$, (b) $L / D=2$, (c) $L / D=1$, (d) $L / D=0.5$, (e) $L / D=0.2$, (f) $L / D=0.1$.

\section{B. Effects of curvature of inlet corner}

Looking at inviscid theory, the pressure becomes unbounded at the sharp corner and the strain rate as well. However, in real situations because of existence of boundary layer and no-slip condition and also separation of flow at sharp corners all parameters remain finite. But still it is expected that the behavior of the flow will be very dependent of the geometry of the corner. Here we will look at flow in nozzles with different radii of curvature from at the inlet corner. $r / D$ is varied between 0.01 and 0.04 while keeping other parameters of the flow and domain constant. Figure 8 shows the threshold value of $K$ versus $r / D$. For both Reynolds numbers of 1000 and 2000, the $K_{t h}$ increases as the $r / D$ increases. This is expected because the larger the radius of curvature, the smaller the increase in velocity and drop in pressure.

\section{Hydraulic flip and its effects on cavitation}

It has been seen that for Reynolds number of 1000 and above flow separates from the wall of the nozzle at the upstream curved corner. This separation creates a recirculation region which extends downstream of the orifice as Reynolds number increases. If this recirculation reaches the downstream corner, then it will merge with the entrained flow outside of orifice, causing the air to enter the orifice and fill the recirculating region. 


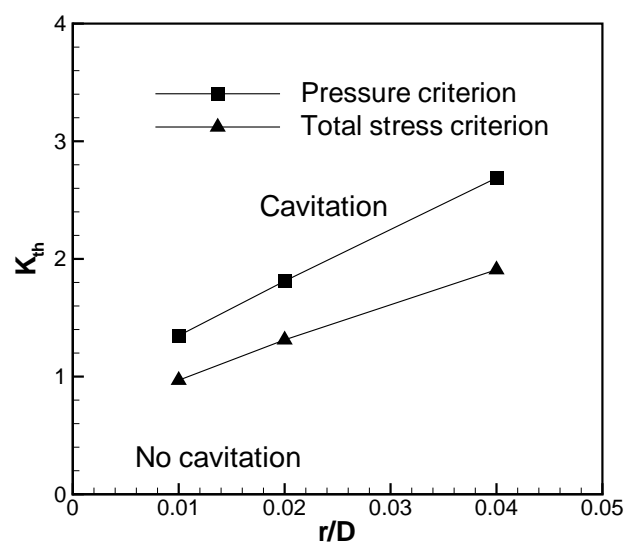

(a) $R e=1000$

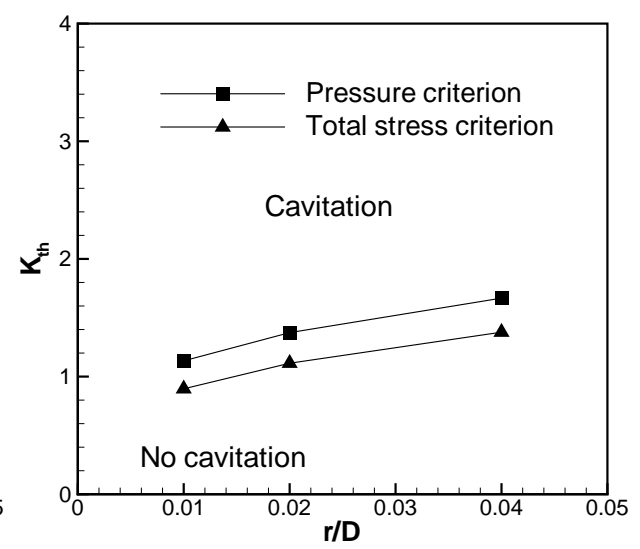

(b) $R e=2000$

Figure 8: Threshold values of $K$ above which cavitation occurs in the orifice. $L / D=2$, (a) $R e=1000$, (b) $R e=2000$.

In order to observe this phenomenon we need higher Reynolds number or shorter nozzles. For diameterto-length ratio of 0.5 , the hydraulic flip happens between Reynolds number of 1000 and 2000. The streamlines for the flow with $R e=2000$ are shown in figure $9 \mathrm{a}$. The thicker line is the liquid-gas interface. Since the flow inside the nozzle reaches a steady state, the streamlines and material lines will be the same and the interface will be a streamline as well. The threshold value of $K$ for this geometry is shown in figure $9 \mathrm{~b}$. The behavior of threshold value of $K$ versus Reynolds number is similar to the results for lower Reynolds number, i.e., $K$ decreases as Reynolds number increases and the total stress criterion predicts a smaller $K$. However, for Reynolds number of 2000, where hydraulic flip happens, the value of $K$ jumps to a higher value which indicates that the cavitation has less chance to occur. This phenomenon has been observed in experiments as well ${ }^{3,22}$ and could be explained by the fact that the air entering the recirculating region will keep the pressure close to downstream pressure.

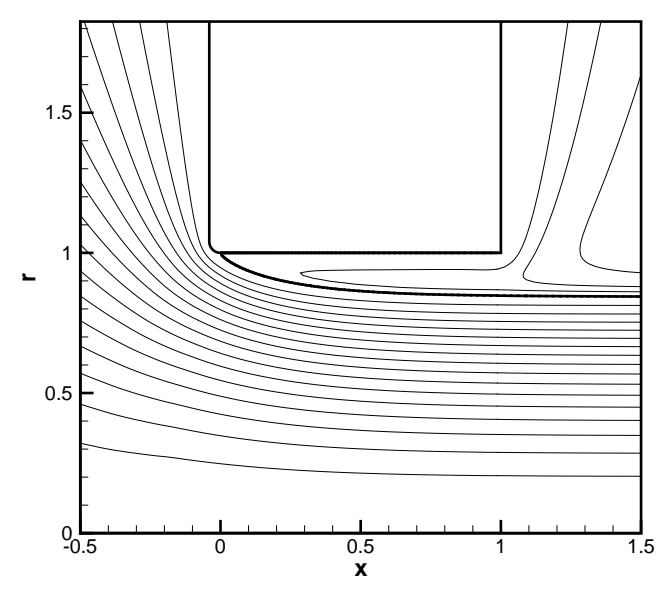

(a)

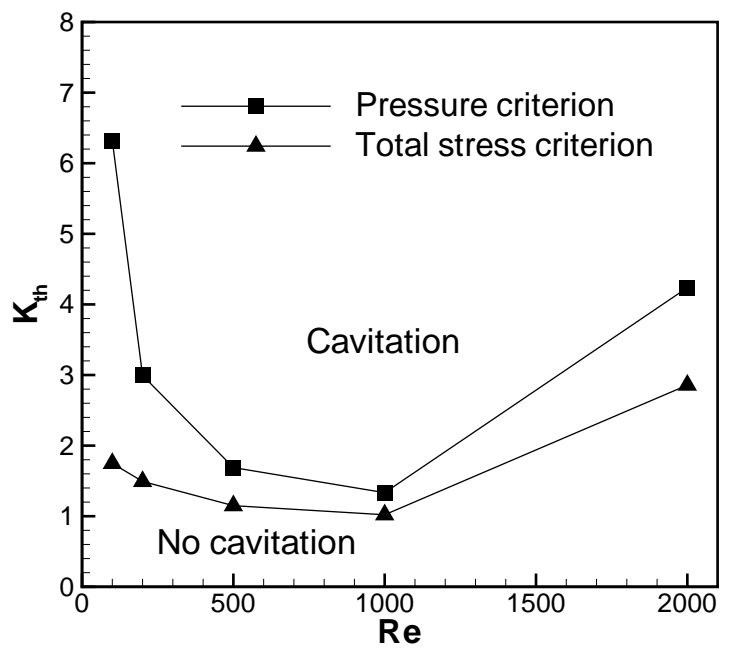

(b)

Figure 9: (a) Streamlines for flow with $R e=2000, L / D=0.5$ (b) Threshold values of $K$ for which cavitation occurs in the orifice for $L / D=0.5$. 


\section{Effect of Weber number on cavitation}

Effect of the Weber number on the cavitation is studied for the flow with Reynolds number of 1000 and orifice with length-to-diameter ratio of two. Threshold values of cavitation number is plotted in figure 10 for Weber numbers of 100, 200, 500, and 1000. As it can be seen in figure 10, the threshold value of $K$ is almost constant for Weber numbers of 500 and above and increases as the Weber number decreases. This could be explained as a consequence of higher pressure difference, for lower Weber number, applied by the surface tension to the liquid inside the jet and the nozzle. The effect of surface tension for $W e>500$ is insignificant for the internal flow and near downstream jet; it probably becomes more significant further downstream.

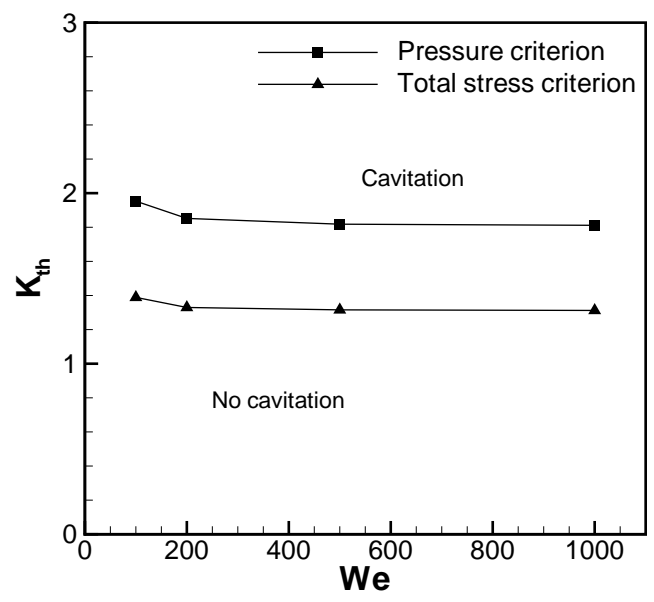

(a)

Figure 10: Threshold values of $K$ for which cavitation occurs in the orifice for $R e=1000, L / D=2.0$ and different Weber numbers.

\section{Conclusions}

The viscous incompressible axisymmetric flow of a liquid through an orifice has been simulated in order to compare the cavitation predicted by the pressure criterion and the total-stress criterion. The total-stress criterion predicts larger cavitating regions in flow field and also lower threshold value of $K$, cavitation number, for occurrence of cavitation relative to the pressure criterion. The quantitative comparison justifies the necessity of new models for cavitation based on the total-stress criterion. The hydraulic flip phenomenon is also observed for flows with high Reynolds number through orifices with small length-to-diameter ratios. It has been observed that when hydraulic flip occurs the cavitating region will shrink or even disappear which is in agreement with experimental observations.

\section{References}

${ }^{1}$ Tamaki, N., Shimizu, M., Nishida, K., and Hiroyasu, H., "Effects of cavitation and internal flow on atomization of a liquid jet," Atomization and Sprays, Vol. 8, No. 2, 1998, pp. 179-197.

${ }^{2}$ Tamaki, N., Shimizu, M., and Hiroyasu, H., "Enhancement of the atomization of a liquid jet by cavitation in a nozzle hole," Atomization and Sprays, Vol. 11, No. 2, 2001, pp. 125.

${ }^{3}$ Hiroyasu, H., "Spray breakup mechanism from the hole-type nozzle and its applications," Atomization and Sprays, Vol. 10, No. 3-5, 2000, pp. 511-527.

${ }^{4}$ Otendal, M., Hemberg, O., Tuohimaa, T. T., and Hertz, H. M., "Microscopic high-speed liquid-metal jets in vacuum," Experiments in Fluids, Vol. 39, No. 5, 2005, pp. 799-804.

${ }^{5}$ Bunnell, R. A., Heister, S. D., Yen, C., and Collicott, S. H., "Cavitating injector flows: Validation of numerical models and simulations of pressure atomizers," Atomization and Sprays, Vol. 9, No. 5, 1999, pp. 445-465.

${ }^{6}$ Tafreshi, H. and Pourdeyhimi, B., "Simulating cavitation and hydraulic flip inside hydroentangling nozzles," Textile Research Journal, Vol. 74, No. 4, 2004, pp. 359-364. 
${ }^{7}$ Ahn, K., Kim, J., and yoon, Y., "Effects of orifice internal flow on transverse injection into subsonic crossflows: Cavitation and hydraulic flip," Atomization and Sprays, Vol. 16, No. 1, 2006, pp. 15-34.

${ }^{8}$ Jung, K., Khil, T., and Yoon, Y., "Effects of orifice internal flow on breakup characteristics of like-doublet injectors," Journal of Propulsion and Power, Vol. 22, No. 3, 2006, pp. 653-660.

${ }^{9}$ Ganippa, L. C., Bark, G., Andersson, and Chomiak, J., "Cavitation: a contributory factor in the transition from symmetric to asymmetric jets in cross-flow nozzles," Experiments in Fluids, Vol. 36, 2004, pp. 627-634.

${ }^{10}$ Joseph, D. D., "Cavitation and the State of Stress in a Flowing Liquid," Journal of Fluid Mechanics, Vol. 284, 1998, pp. 367-376.

${ }^{11}$ Funada, T., Wang, J., and Joseph, D. D., "Viscous potential flow analysis of stress induced cavitation in an aperture flow," Atomization and Sprays, Vol. 16, No. 7, 2006, pp. 763-776.

${ }^{12}$ Dabiri, S., Sirignano, W. A., and Joseph, D. D., "Two-Dimensional Viscous Aperture Flow: Navier-Stokes and ViscousPotential-Flow Solutions," In preparation.

${ }^{13}$ J. F. Thompson, and F. C. Thames, a. C. W. M., "Automatic numerical generation of body-fitted curvilinear coordinate system for field containing any number of arbitrary 2-dimensional bodies," Journal of Computational Physics, Vol. 15, No. 3, 1974, pp. 299-319.

${ }^{14}$ Ryskin, G. and Leal, L., "Orthogonal mapping," Journal of Computational Physics, Vol. 50, No. 1, 1983, pp. 71-100.

${ }^{15}$ Hayase, T., Humphrey, J. A. C., and Greif, R., "A consistently formulated QUICK scheme for fast and stable convergence using finite-volume iterative calculation procedure," J. Comput. Phys., Vol. 98, 1992, pp. 108-118.

${ }^{16}$ Patankar, S. V., Numerical heat transfer and fluid flow, Hemisphere, Washington, DC/New York, 1980.

${ }^{17}$ Sussman, M., Fatemi, E., Smereka, P., and Osher, S., "An improved level set method for incompressible two-phase flows," Computers and Fluids, Vol. 27, No. 5-6, 1998, pp. 663-680.

${ }^{18}$ Osher, S. and Fedkiw, R. P., "Level set methods: an overview and some recent results," J. Comput. Phys., Vol. 169, 2001, pp. 436

${ }^{19}$ Pan, Y. and Suga, K., "A numerical study on the breakup process of laminar liquid jets into a gas," Physics of Fluids, Vol. 18, No. 5, 2006, Art. No. 052101.

${ }^{20}$ Rupe, J. H., "On the dynamic characteristics of free liquid jets and a partial correlation with orifice geometry," NASA Technical Report, , No. 32207, 1962.

${ }^{21}$ Padrino, J. C., Joseph, D. D., Funada, T., Wang, J., and Sirignano, W. A., "Stress-induced cavitation for the streaming motion of a viscous liquid past a sphere," Journal of Fluid Mechanics, 2007, in press.

${ }^{22}$ Chaves, H., Knapp, M., Kubitzek, A., Obermeier, F., and Schneider, T., "Experimental study of cavitation in the nozzle hole of diesel injectors using transparent nozzles." SAE papers, , No. 1995-0290, 1995. 